MATHEMATICS OF COMPUTATION

Volume 76, Number 259, July 2007, Pages 1661-1668

S $0025-5718(07) 01969-2$

Article electronically published on January 25, 2007

\title{
CONSTRUCTING REPRESENTATIONS OF HIGHER DEGREES OF FINITE SIMPLE GROUPS AND COVERS
}

\author{
VAHID DABBAGHIAN-ABDOLY
}

\begin{abstract}
Let $G$ be a finite group and $\chi$ an irreducible character of $G$. A simple method for constructing a representation affording $\chi$ can be used whenever $G$ has a subgroup $H$ such that $\chi_{H}$ has a linear constituent with multiplicity 1. In this paper we show that (with a few exceptions) if $G$ is a simple group or a covering group of a simple group and $\chi$ is an irreducible character of $G$ of degree between 32 and 100, then such a subgroup exists.
\end{abstract}

\section{INTRODUCTION}

Let $G$ be a finite group and $\chi$ be an irreducible character of $G$. We say that a subgroup $H$ is a $\chi$-subgroup if the restriction $\chi_{H}$ of $\chi$ to $H$ has at least one linear constituent of multiplicity 1. Not every pair $(\chi, G)$ has a $\chi$-subgroup (see [10] and Section 4 below), but $\chi$-subgroups can be found in many cases. The existence of such subgroups is of interest for several reasons such as computing primitive idempotent elements (see [10, 9] and [15]), calculating Schur indices (see [8], 14] and [16) and computing irreducible matrix representations (see [6], 5] and [4]).

A simple and efficient method for computing matrix representations of finite groups has been described by Dixon [6]. This applies whenever $G$ has a $\chi$-subgroup for an irreducible character $\chi$ and works best when the subgroup is small. In practice, the difficulty in using this method lies in finding such a $\chi$-subgroup (if one exists). This could involve searching through the full lattice of the subgroup of $G$ and that becomes impractical for large groups.

In 4 the author developed a general program to compute a matrix representation affording any specified character $\chi$ of a group $G$ (see also the GAP package [5]). The program uses a recursive technique which reduces the general problem to the special problem of computing representations affording irreducible characters of degree at most $\chi(1)$ for central covers of simple groups.

Thus we must deal with the problem of computing a representation in the case where $\chi$ is irreducible and $G$ is simple or a cover of a simple group. In [4] (see also [3]) the author shows that in this special case we can usually find a suitable $\chi$-subgroup when $\chi(1)<32$. In a few cases where we failed to find $\chi$-subgroups for characters in this range we found a maximal subgroup $M$ of $G$ such that $\chi_{M}$ is irreducible. In the latter case a representation of $M$ affording $\chi_{M}$ can be constructed (recursively), and we showed how to extend this representation of $M$ to

Received by the editor November 27, 2005 and, in revised form, July 6, 2006.

2000 Mathematics Subject Classification. Primary 20C40; Secondary $20 \mathrm{C} 15$.

Key words and phrases. Simple group, central cover, irreducible representation.

This work was supported by the MITACS NCE and NSERC of Canada.

(C)2007 American Mathematical Society Reverts to public domain 28 years from publication 
a representation of $G$. Thus our general program can be applied to construct a representation affording a character of degree $<32$ for any group.

In the present paper we extend the result of $[3]$ (and hence the capability of the original program) to characters with degrees between 32 and 100. Again we shall show that, in most cases, if $\chi$ is an irreducible character of a cover $G$ of a simple group and $32 \leqslant \chi(1) \leqslant 100$, then we can find a $\chi$-subgroup which is easily described in terms of the group structure (see Section 2). In the remaining cases, with two exceptions, we find a maximal subgroup $M$ of $G$ such that $\chi_{M}$ is irreducible (see Section 3). The exceptions occur for the cover $6 . A_{7}$ and two characters of degree 36 ; in these two cases there are neither $\chi$-subgroups nor irreducible maximal subgroups available, so a different approach will be needed to construct the corresponding matrix representations for these characters (see Section 4).

\section{2. $\chi$-SUBGROUPS}

The following theorem describes $\chi$-subgroups for certain irreducible characters $\chi$ of alternating groups. For a proof see [4].

Theorem 2.1. Let $\chi$ be an irreducible character of the alternating group $A_{n}$.

(1) If $n \geqslant 4$ and $\chi(1)=n-1$, then a Sylow 3-subgroup of order 3 of $A_{4}$ is a $\chi$-subgroup.

(2) If $n \geqslant 6$ and $\chi(1)=(n-1)(n-2) / 2$ or $n(n-3) / 2$, then a Sylow 3-subgroup of $A_{6}$ of order 9 is a $\chi$-subgroup.

(3) If $n \geqslant 8$ and $\chi(1)=(n-1)(n-2)(n-3) / 6, n(n-1)(n-5) / 6$ or $n(n-$ $2)(n-4) / 3$, then a Sylow 2-subgroup of $A_{8}$ of order 64 is a $\chi$-subgroup.

With the exception of the characters covered in the theorem above, there are only a few cases where an alternating group has an irreducible character of degree between 32 and 100. In these cases a $\chi$-subgroup was computed directly using GAP [7. These exceptions are listed in Table 1. This table also contains $\chi$-subgroups for the covering groups of alternating groups and some other simple groups and covers listed in the Atlas [1] (see also [2]). For these groups there is no general theorem about their $\chi$-subgroups when $32 \leqslant \chi(1) \leqslant 100$.

The group $\operatorname{SL}(2, q)$ is the unique covering group of the simple group $\operatorname{PSL}(2, q)$, except for $q=9$. In the latter case $\operatorname{PSL}(2,9) \cong A_{6}$. By [13, Theorem 7.1.1] the group $\mathrm{SL}(3, q)$ where $q>2$, is the unique covering group of the simple group $\operatorname{PSL}(3, q)$ except when $q=4$ (the group $\operatorname{PSL}(3,4)$ has 7 different covering groups, see Table 1$)$. Also $\operatorname{PSU}(3, q)$ is a simple group of twisted Lie type ${ }^{2} A_{2}(q)$ and the group $\mathrm{SU}(3, q)$ is the unique covering group of the simple group $\operatorname{PSU}(3, q)$ (see [11, Corollary 5.1.3]).

On the other hand, $\operatorname{PSL}(2, q), \operatorname{PSL}(3, q)$ and $\operatorname{PSU}(3, q)$ are the factor groups of $\mathrm{SL}(2, q), \mathrm{SL}(3, q)$ and $\mathrm{SU}(3, q)$ by their centres, so characters of the former groups correspond to those characters of $\mathrm{SL}(2, q), \mathrm{SL}(3, q)$ and $\mathrm{SU}(3, q)$, respectively, whose kernels contain the centre. Thus it is enough to find $\chi$-subgroups for the irreducible characters $\chi$ of $\mathrm{SL}(2, q), \mathrm{SL}(3, q)$ and $\mathrm{SU}(3, q)$ (except for $\operatorname{PSL}(3,4)$ and covers). The following theorems describe these $\chi$-subgroups. There is no restriction on the degree of the characters. For a proof of these theorems see 4 .

Theorem 2.2. Let $G=\operatorname{SL}(2, q)(G=\mathrm{SL}(3, q))$ where $q>4(q>2)$ is a power of a prime $p$ and let $H$ be a Sylow p-subgroup of $G$. Then $H$ is a $\chi$-subgroup for all irreducible characters $\chi$ of $G$. 
Theorem 2.3. Let $G=\mathrm{SU}(3, q)$ where $q>2$ is a power of a prime $p$ and let $H$ be a Sylow p-subgroup of $G$. Then $H$ is a $\chi$-subgroup for all irreducible characters $\chi$ of $G$ such that $\chi(1) \neq q^{2}-q$. If $\chi(1)=q^{2}-q$, then $H$ contains an abelian subgroup of order $q^{2}$ which is a $\chi$-subgroup.

Let $G$ be a group and $\chi$ a character of $G$. We denote by $\operatorname{Lin}(\chi)$ and $\operatorname{Lin}(G)$ the set of linear constituents of $\chi$ and the set of linear characters of $G$, respectively. Clearly $\operatorname{Lin}(\chi) \subseteq \operatorname{Lin}(G)$. The following simple remark is very useful.

Remark 2.4. Let $\chi$ be an irreducible character of $G$ and $H$ a Sylow subgroup of $G$. Let $\mu$ be a character of $H$ such that $\chi(1)=|H|+\mu(1)$ and $\chi_{H}(h)=\mu(h)$ for all $1 \neq h \in H$. Then $\operatorname{Lin}(\mu) \neq \operatorname{Lin}(H)$ implies $H$ is a $\chi$-subgroup. This is true because the hypotheses show that $\chi_{H}=\rho+\mu$ where $\rho$ is the regular character of $H$. Since $\operatorname{Lin}(\mu) \subset \operatorname{Lin}(\rho)=\operatorname{Lin}(H)$, there exists a linear character $\varphi$ of $H$ such that $\left\langle\chi_{H}, \varphi\right\rangle=1$.

We now examine each case in the Atlas [1] where $G$ is a simple group or the cover of a simple group and $\chi$ is an irreducible character of degree between 32 and 100, and which was not covered by Theorems 2.1, 2.2 and 2.3. Using GAP [7, the remark above and the information available from [1] we searched for a subgroup of $G$ of a simply described form which is a $\chi$-subgroup. We found that in most cases such $\chi$-subgroups exist.

The group libraries in GAP and 2] are used as the sources for generators of the groups listed in Tables 1 and 2. For finding most of the $\chi$-subgroups listed in these tables we used the available standard functions in GAP. For each group $G$ in Table 1 we computed Sylow $r$-subgroups, say $P_{r}$, and some simply relative subgroups to $P_{r}$ such as the derived subgroup $P_{r}^{\prime}$ and the normalizers and centralizers of $P_{r}$ and $P_{r}^{\prime}$ in $G$. Then we found the smallest possible $\chi$-subgroup among these subgroups. The only exception in Table 1 is an irreducible character of degree 32 of 2.M12. In this case we found a particular 2-subgroup which is denoted by $H^{*}$. These results are summarized in Table 1.

TABLE 1 . The $\chi$-subgroups of simple groups and covers for $32 \leqslant$ $\chi(1) \leqslant 100$

\begin{tabular}{|l||l|l|l|}
\hline Group & $\chi(1)$ & $\chi$-subgroup & Size of $\chi$-subgroup \\
\hline \hline$A_{7}$ & 35 & $N_{G}\left(P_{5}\right)$ & 20 \\
\hline $2 . A_{7}$ & 36 & $N_{G}\left(P_{3}\right)$ & 72 \\
\hline$M_{11}$ & 44,45 & $N_{G}\left(P_{5}\right)$ & 20 \\
& 55 & $N_{G}\left(P_{11}\right)$ & 55 \\
\hline$A_{8}$ & $45,56,70$ & $N_{G}\left(P_{5}\right)$ & 60 \\
\hline $2 . A_{8}$ & $48,56,64$ & $N_{G}\left(P_{5}\right)$ & 120 \\
\hline $2 \cdot L_{3}(4)$ & $36,64,70,90$ & $N_{G}\left(P_{3}\right)$ & 144 \\
\hline $4_{1} \cdot L_{3}(4)$ & $56,64,80$ & $N_{G}\left(P_{3}\right)$ & 288 \\
\hline $4_{2} \cdot L_{3}(4)$ & 36 & $N_{G}\left(P_{3}\right)$ & 288 \\
\hline$U_{4}(2)$ & $40,60,64,81$ & $P_{2}$ & 64 \\
& 45 & $N_{G}\left(P_{5}\right)$ & 20 \\
\hline $2 \cdot U_{4}(2)$ & $36,60,64,80$ & $P_{3}$ & 81 \\
\hline
\end{tabular}


(TABLE 1. continued)

\begin{tabular}{|l||l|l|l|}
\hline$S z(8)$ & 35 & $N_{G}\left(P_{7}\right)$ & 14 \\
& $64,65,91$ & $N_{G}\left(P_{13}\right)$ & 52 \\
\hline $2 . S z(8)$ & $40,56,64$ & $N_{G}\left(P_{13}\right)$ & 104 \\
\hline$M_{12}$ & $45,54,55,66$ & $N_{G}\left(P_{5}\right)$ & 40 \\
& 99 & $N_{G}\left(P_{11}\right)$ & 55 \\
\hline $2 . M_{12}$ & 32 & $H^{*}$ & 64 \\
& 44 & $P_{3}$ & 27 \\
\hline$J_{1}$ & $56,76,77$ & $N_{G}\left(P_{7}\right)$ & 42 \\
\hline$A_{9}$ & $35,42,84$ & $N_{G}\left(P_{7}\right)$ & 42 \\
\hline $2 . A_{9}$ & 48,56 & $N_{G}\left(P_{7}\right)$ & 84 \\
\hline$M_{22}$ & 45,55 & $N_{G}\left(P_{5}\right)$ & 20 \\
& 99 & $N_{G}\left(P_{11}\right)$ & 55 \\
\hline $2 . M_{22}$ & 56 & $N_{G}\left(P_{5}\right)$ & 40 \\
\hline $3 . M_{22}$ & 45 & $N_{G}\left(P_{5}\right)$ & 60 \\
& 99 & $P_{2}$ & 128 \\
\hline $4 . M_{22}$ & 56 & $N_{G}\left(P_{3}\right)$ & 288 \\
\hline$J_{2}$ & $63,70,90$ & $N_{G}\left(P_{7}\right)$ & 42 \\
& 36 & $P_{5}$ & 25 \\
\hline $2 . J_{2}$ & $50,56,64$ & $N_{G}\left(P_{7}\right)$ & 84 \\
& 84 & $N_{G}\left(P_{3}\right)$ & 432 \\
\hline$S_{4}(4)$ & 34,51 & $P_{5}$ & 25 \\
& 50 & $N_{G}\left(P_{3}\right)$ & 72 \\
& 85 & $P_{2}$ & 256 \\
\hline$S_{6}(2)$ & $35,56,70,84$ & $N_{G}\left(P_{7}\right)$ & 42 \\
\hline $2 . S_{6}(2)$ & 48,64 & $P_{3}$ & 81 \\
\hline$A_{10}$ & 42 & $P_{5}$ & 25 \\
& 90 & $P_{3}$ & 81 \\
\hline $2 . A_{10}$ & 48,64 & $P_{3}$ & 81 \\
\hline$U_{4}(3)$ & 35 & $N_{G}\left(P_{5}\right)$ & 20 \\
& 90 & $C_{G}\left(P_{3}^{\prime}\right)$ & 81 \\
\hline $2 . U_{4}(3)$ & 56 & $N_{G}\left(P_{5}\right)$ & 40 \\
& 70 & $C_{G}\left(P_{3}^{\prime}\right)$ & 162 \\
\hline $3{ }_{2} . U_{4}(3)$ & 36,45 & $N_{G}\left(P_{5}\right)$ & 60 \\
\hline $6_{1} . U_{4}(3)$ & 84 & $C_{G}\left(P_{3}^{\prime}\right)$ & 486 \\
\hline $12_{1} . U_{4}(3)$ & 84 & $C_{G}\left(P_{3}^{\prime}\right)$ & 972 \\
\hline $6_{2} . U_{4}(3)$ & 90 & $N_{G}\left(P_{5}\right)$ & 120 \\
\hline $12_{2} . U_{4}(3)$ & 36 & $N_{G}\left(P_{5}\right)$ & 240 \\
\hline$G_{2}(3)$ & $64,78,91$ & $N_{G}\left(P_{7}\right)$ & 42 \\
\hline$S_{4}(5)$ & 40 & $N_{G}\left(P_{13}\right)$ & 52 \\
& 65,78 & $P_{2}$ & 64 \\
& 90 & $C_{G}\left(P_{5}^{\prime}\right)$ & 125 \\
\hline $2 . S_{4}(5)$ & 52 & $\left.P_{13}\right)$ & 104 \\
\hline
\end{tabular}


(TABLE 1. continued)

\begin{tabular}{|l||l|l|l|}
\hline$L_{4}(3)$ & 39 & $P_{3}^{\prime}$ & 27 \\
& 52 & $N_{G}\left(P_{5}\right)$ & 80 \\
& 65 & $N_{G}\left(P_{13}\right)$ & 39 \\
& 90 & $C_{G}\left(P_{3}^{\prime}\right)$ & 81 \\
\hline $2 . L_{4}(3)$ & 40 & $P_{3}^{\prime}$ & 27 \\
\hline$M_{23}$ & 45 & $P_{23}$ & 23 \\
\hline$U_{5}(2)$ & $44,55,66$ & $N_{G}\left(P_{11}\right)$ & 55 \\
\hline${ }^{2} F_{4}(2)^{\prime}$ & 78 & $N_{G}\left(P_{13}\right)$ & 78 \\
\hline$H S$ & 77 & $N_{G}\left(P_{11}\right)$ & 55 \\
\hline $2 . H S$ & 56 & $N_{G}\left(P_{7}\right)$ & 84 \\
\hline$J_{3}$ & 85 & $P_{3}^{\prime}$ & 27 \\
\hline$O_{8}^{+}(2)$ & $35,50,84$ & $N_{G}\left(P_{7}\right)$ & 42 \\
\hline $2 . O_{8}^{+}(2)$ & 56 & $N_{G}\left(P_{7}\right)$ & 84 \\
\hline$O_{8}^{-}(2)$ & 34,84 & $P_{3}$ & 81 \\
& 51 & $N_{G}\left(P_{17}\right)$ & 68 \\
\hline${ }^{3} D_{4}(2)$ & 52 & $P_{7}$ & 49 \\
\hline$M_{24}$ & 45 & $P_{23}$ & 23 \\
\hline$G_{2}(4)$ & 65 & $N_{G}\left(P_{13}\right)$ & 78 \\
& 78 & $P_{2}^{\prime}$ & 256 \\
\hline$H e$ & 51 & $P_{3}$ & 27 \\
\hline$O_{7}(3)$ & 78 & $P_{2}^{\prime}$ & 32 \\
& 91 & $N_{G}\left(P_{13}\right)$ & 78 \\
\hline$S_{6}(3)$ & 78 & $N_{G}\left(P_{7}\right)$ & 84 \\
& 91 & $C_{G}\left(P_{3}^{\prime}\right)$ & 27 \\
\hline $2 . U_{6}(2)$ & 56 & $P_{3}$ & 729 \\
\hline$S_{8}(2)$ & 35 & $C_{G}\left(P_{2}^{\prime}\right)$ & 16 \\
& 51,85 & $N_{G}\left(P_{17}\right)$ & 136 \\
\hline $3 . S u z$ & 66,78 & $P_{3}^{\prime}$ & 243 \\
\hline $2 . F_{4}(2)$ & 52 & $P_{7}$ & 49 \\
\hline & & & \\
\end{tabular}

Among the remaining cases, not covered in Table 1, a direct search showed that there were $\chi$-subgroups of a more complicated form. These are summarized in Table 2 , and the $\chi$-subgroups are described in more detail as follows.

TABLE 2. The $\chi$-subgroups of exceptional characters

\begin{tabular}{|l||l|l|}
\hline Group & $\chi(1)$ & Size of $\chi$-subgroup \\
\hline \hline $6 . L_{3}(4)$ & $36,42,60$ & 1008 \\
& 90 & 60 \\
\hline $12_{1} \cdot L_{3}(4)$ & 48 & 720 \\
\hline $12_{2} \cdot L_{3}(4)$ & 36 & 288 \\
& $48,60,84$ & 720 \\
\hline $6 . M_{22}$ & 66 & 330 \\
\hline$F i_{22}$ & 78 & 32 \\
\hline
\end{tabular}


If $G=6 . L_{3}(4)$, then $G$ has a maximal subgroup $H$ of order 1008 such that $H / Z(G) \cong L_{2}(7)$. The subgroup $H$ is a $\chi$-subgroup for irreducible characters $\chi$ of $G$ of degrees 36,42 and 60 . For $\chi(1)=90$ the group $G$ has a maximal subgroup $M$ such that $M / Z(G) \cong A_{6}$. Then $M$ contains a normal subgroup $H$ of order 60 such that $H \cong A_{5}$ and is a $\chi$-subgroup.

If $G=12_{1} \cdot L_{3}(4)$, then $G$ has a maximal subgroup $M$ such that $M / Z(G) \cong A_{6}$. The subgroup $M$ contains a maximal subgroup $H$ of order 720 such that $H / Z(G) \cong$ $A_{5}$ and is a $\chi$-subgroup for $\chi(1)=48$.

If $G=12_{2} \cdot L_{3}(4)$, then $G$ has a maximal subgroup $M$ such that $M / Z(G) \cong$ $L_{2}(7)$. The subgroup $M$ contains a maximal subgroup $H$ of order 288 such that $H / Z(G) \cong S_{4}$ and is a $\chi$-subgroup for $\chi(1)=36$. Also the group $G$ has a maximal subgroup $M$ such that $M / Z(G) \cong A_{6}$. Then $M$ contains a maximal subgroup $H$ of order 720 such that $H / Z(G) \cong A_{5}$ and is a $\chi$-subgroup for $\chi(1) \in\{48,60,84\}$.

If $G=6 . M_{22}$, then $G$ has a maximal subgroup $M$ such that $M / Z(G) \cong L_{2}(11)$. The subgroup $M$ contains a maximal subgroup $H$ of order 330 which is a $\chi$-subgroup for both of the characters of degree 66 .

Finally if $G=F i_{22}$ and $\chi$ is an irreducible character of $G$ of degree 78 , then $G$ contains a maximal subgroup $M \cong O_{7}(3)$ such that $\chi_{M}$ is irreducible. Now using Table 1 we see that the derived subgroup of order 32 of a Sylow 2-subgroup of $M$ is a $\chi$-subgroup.

\section{Maximal subgroups}

There are three groups in 1 with characters of degree $\leqslant 100$ for which we do not know whether there are $\chi$-subgroups in these cases. These were the covering groups 2. $A_{12}, 2 . A_{13}$ and $6 . A_{7}$. We shall consider $6 . A_{7}$ in the next section. For the first two groups and each of their characters $\chi$ of degree $\leqslant 100$ we shall find a maximal subgroup $M$ such that $\chi_{M}$ is irreducible (see the Introduction). To do this we use the technique described in [3].

If $G=A_{12}$, then the covering group 2.G has an irreducible character $\chi$ of degree 32 , and $G$ has a maximal subgroup $M \cong\left(A_{6} \times A_{6}\right): 2^{2}$ of index 462 such that $\chi_{\tilde{M}}$ is irreducible. If we take $H$ a subgroup of $G$ isomorphic to $A_{6}$ and $K$ the normalizer of $H$ in $G$, then $M$ is the normalizer of $K$ in $G$ (i.e., $M=N_{G}\left(N_{G}(H)\right)$ ).

For $G=A_{13}$, the covering group 2.G has two irreducible characters $\chi$ of degree 32. In this case $G$ has a maximal subgroup $M$ of index 13 such that $M \cong A_{12}$ and $\chi_{\tilde{M}}$ is irreducible.

\section{Exceptional Characters of $6 \cdot A_{7}$}

Finally we show that if $\tilde{G}=6 . A_{7}$ and $\chi$ is one of the two faithful irreducible characters of $\tilde{G}$ of degree 36 , then $\chi_{\tilde{M}}$ is not irreducible for any maximal subgroup $\tilde{M}$ of $\tilde{G}$, and $\tilde{G}$ has no $\chi$-subgroups.

If $\tilde{M}_{i}$ is a maximal subgroup of $\tilde{G}$, then $\tilde{M}_{i} / Z(\tilde{G})$ is isomorphic to one of the groups $\left(A_{4} \times 3\right): 2, \operatorname{PSL}(2,7), S_{5}$ and $A_{6}$ for $i=1,2,3$ and 4 , respectively (see [1]). It is straightforward to check that $\chi_{\tilde{M}_{i}}$ is not irreducible for any maximal subgroup $\tilde{M}_{i}$ of $\tilde{G}$.

A direct method to show that $\tilde{G}$ has no $\chi$-subgroups, is to search among all nonconjugate subgroups of $\tilde{G}$ to see that the restriction of $\chi$ to all of these subgroups has no linear constituents of multiplicity 1 . 
Another approach is the restriction of $\chi$ to maximal subgroups, as follows. Since $\chi_{\tilde{M}_{1}}=3 \theta_{1}+3 \theta_{2}$ where $\theta_{1}$ and $\theta_{2}$ are irreducible characters of $\tilde{M}_{1}$ of degree $6, \tilde{M}_{1}$ does not contain any $\chi$-subgroups. Furthermore, $\chi_{\tilde{M}_{i}}$ has no linear constituents for $i=2,3,4$. Now we restrict $\chi$ to the maximal subgroups $\tilde{M}_{i}$ for $i=2,3,4$.

The group $\tilde{M}_{3}$ has 4 non-conjugate maximal subgroups. Let $\tilde{M}_{34}$ be a maximal subgroup of $\tilde{M}_{3}$ of index 3 . Then $\tilde{M}_{34} \cong \mathrm{SL}(2,7)$ and it is the only maximal subgroup of $\tilde{M}_{3}$ such that $\chi_{\tilde{M}_{34}}$ has some constituents of multiplicity 1 . Since these constituents are not linear, $\tilde{M}_{34}$ is not a $\chi$-subgroup but, if $\tilde{M}_{3}$ contains a $\chi$-subgroup, it must be a subgroup of $\tilde{M}_{34}$. Straightforward computations show that the multiplicities of the constituents of the restriction of $\chi$ to all maximal subgroups of $\tilde{M}_{34}$ are larger than 1 . This implies that $\tilde{M}_{34}$ and, in turn, $\tilde{M}_{3}$ does not contain any $\chi$-subgroups.

A similar argument holds for $\tilde{M}_{2}$ and $\tilde{M}_{4}$. The group $\tilde{M}_{2}$ has two maximal subgroups $\tilde{M}_{24}$ and $\tilde{M}_{25}$ of indices 2 and 3 , respectively, such that $\tilde{M}_{24} / Z(\tilde{G}) \cong S_{5}$ and $\tilde{M}_{25} / Z(\tilde{G}) \cong A_{5}$. Also $\tilde{M}_{4}$ contains two maximal subgroups $\tilde{M}_{44}$ and $\tilde{M}_{45}$ of index 6 such that $\tilde{M}_{44} / Z(\tilde{G}) \cong \tilde{M}_{45} / Z(\tilde{G}) \cong A_{5}$. These are the only maximal subgroups of $\tilde{M}_{2}$ and $\tilde{M}_{4}$ such that the restriction of $\chi$ has some constituents of multiplicity 1 . Since none of these constituents are linear, if $\tilde{M}_{2}$ or $\tilde{M}_{4}$ contains a $\chi$ subgroup, it needs to be a subgroup of one of these maximal subgroups. The groups $\tilde{M}_{24}, \tilde{M}_{25}, \tilde{M}_{44}$ and $\tilde{M}_{45}$ contain only one maximal subgroup $\tilde{H} \cong 2 . A_{5}$ such that $\chi_{\tilde{H}}$ has some constituents of multiplicity 1 . It is easy to see that the multiplicities of the constituents of the restriction of $\chi$ to all of the maximal subgroups of $\tilde{H}$ are bigger than 1. Therefore all the maximal subgroups of $\tilde{M}_{2}$ and $\tilde{M}_{4}$ and, in turn, $\tilde{M}_{2}$ and $\tilde{M}_{4}$ have no $\chi$-subgroups. This proves that $\tilde{G}$ has no $\chi$-subgroups.

\section{ACKNOWLEDGMENTS}

The author would like to thank Michael Monagan and the Department of Mathematics of Simon Fraser University for the computer resources and for their generous hospitality. He also wants to thank the reviewers and John Dixon for their careful reading of the manuscript.

\section{REFERENCES}

1. J.H. Conway, R.T. Curtis, S.P. Norton, R.A. Parker and R.A. Wilson, Atlas of Finite Groups. Maximal Subgroups and Ordinary Characters for Simple Groups, Claredon Press, Oxford, 1985. MR 827219 (88g:20025)

2. ATLAS of Finite Group Representations, School of Mathematics and Statistics, The University of Birmingham, Version 2, (http://web.mat.bham.ac.uk/atlas/v2.0/).

3. V. Dabbaghian-Abdoly, Constructing representations of finite simple groups and covers, Canad. J. Math., 58 (2006), 23-38. MR2195590

4. V. Dabbaghian-Abdoly, An algorithm to construct representations of finite groups, Ph.D. thesis, School of Mathematics, Carleton University, 2003.

5. V. Dabbaghian-Abdoly, RPSEN - A Package for Constructing Representations of Finite Groups, GAP Package, 2004, (http://www.gap-system.org/Packages/repsn.html).

6. J.D. Dixon, Constructing representations of finite groups, DIMACS Ser. Discrete Math. Theoret. Comput. Sci. 11, Amer. Math. Soc., Providence, RI (1993), 105-112. MR1235797 (94h:20011)

7. The GAP Group, GAP-Groups, Algorithms, and Programming. Version 4.6 (2005), (http://www.gap-system.org).

8. R. Gow, Schur indices of some groups of Lie type, J. Algebra, 42 (1976), 102-120. MR0466330 $(57: 6210)$ 
9. E. Güzel, Primitive idempotents of the group algebra $\mathbb{C S L}(3, q)$, Math. Scand., 70 (1992), no. 2, 177-185. MR.1189972 (93k:20027)

10. G.J. Janusz, Primitive idempotents in group algebras, Proc. Amer. Math. Soc., 17 (1966), 520-523. MR0194523 (33:2733)

11. D. Gorenstein, R. Lyons and R. Solomon, The Classification of the Finite Simple Groups: Almost Simple K-groups, Number 3., Part I., Amer. Math. Soc., Providence, RI, 1998. MR 1490581 (98j:20011)

12. I.M. Isaacs, Character Theory of Finite Groups, Dover, New York, 1994. MR.1280461

13. G. Karpilovsky, The Schur Multiplier, London Math. Soc. Monographs, Oxford Univ., New York, 1987. MR 1200015 (93j:20002)

14. Z. Ohmori, On a Zelevinsky theorem and the Schur indices of the finite unitary groups, J. Math. Sci. Univ. Tokyo, 4 (1997), 417-433. MR1466354 (98i:20045)

15. N. Yelkenkaya, Primitive idempotents of the group algebra $\mathbb{C S L}(2, q)$, Istanbul Univ. Fen Fak. Mat. Derg., 55/56 (1996/97), 99-109. MR1767540 (2001f:16051)

16. A.V. Zelevinsky, Representations of Finite Classical Groups, Lecture Notes in Mathematics 869, Springer, New York, 1981. MR643482 (83k:20017)

The Centre for Experimental and Constructive Mathematics (CECM), Simon Fraser University, Burnaby, British Columbia, V5A 1S6, Canada

E-mail address: vdabbagh@cecm.sfu.ca 